\title{
Éditorial
}

\section{Le corps en première personne : une écologie pré-motrice}

\section{Body awareness in first person: A pre-motor ecology}

Bernard Andrieu

Ce numéro se propose de réfléchir sur ce qui se produit dans ce temps en avance sur la conscience motrice. Que se passe-t-il dans ces moments pré-moteurs inconscients mais dont nous pressentons par notre sensibilité nerveuse la venue sans pouvoir l'anticiper mentalement?

Le corps est constitué par les techniques incorporées, les gestes habituels et les postures quotidiennes mais notre conscience n'en prend connaissance que lors d'une auto-confrontation en première et deuxième personne ou lors d'une transmission motrice à une troisième personne comme dans l'action ou dans l'enseignement et l'éducation.

Pourtant la mémoire, la sensibilité et la perception $\mathrm{du}$ corps produisent des informations que nous transmettons sans une compréhension consciente. Car le corps vivant s'écrit de lui-même en première personne avant que par l'émersion de ses sensations ressenties par notre conscience ne les traduisent dans un récit par une première personne incarnée (Fig. 1).

Notre attention au monde n'est donc pas entièrement consciente et volontaire. Concevoir l'incarnation comme un mouvement par lequel l'esprit s'incarnerait dans le corps pour se manifester nous prive d'une compréhension sensible et globale de l'activité corporelle. Le corps vivant, plutôt que vécu, est en interaction dynamique avec son environnement dès sa formation, au cours de son développement et dans son activité pré-cognitive. L'écologie pré-motrice est cette interaction sensible et spontanée du corps avec ses environnements

À travers ces articles nous explorons du degré le plus conscient jusqu'au degré le plus inconscient cette écologisation du corps de la pré-motricité à l'attention motrice la plus volontaire (Tab. 1).

En partant de l'attention consciente se dresse la possibilité d'une réflexivité en action de la motricité, Alain Mouchet analyse l'expérience subjective en sport à partir d'un éclairage psycho phénoménologique de l'attention. En considérant l'attention comme une modulation

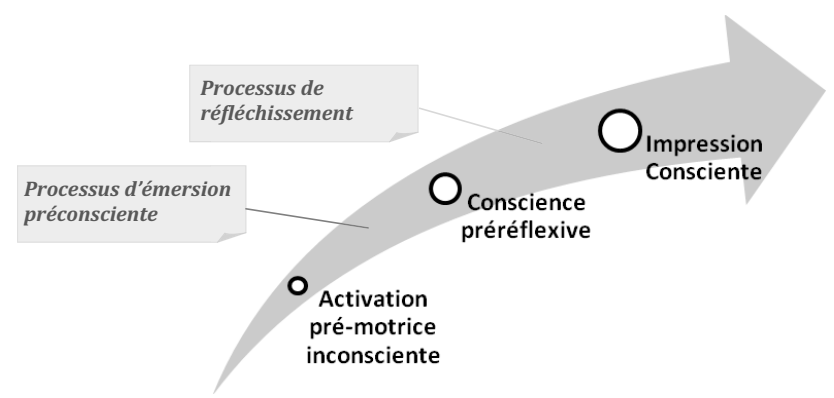

Fig. 1. Modélisation des niveaux d'appréhension de l'expérience vécue et des processus liés à leur conscientisation.

de la conscience, avec une structure complexe et une dynamique attentionnelle, le cadre d'analyse original est susceptible d'éclairer l'activité des sujets en contexte habituel de pratique : «Une telle perspective permet en effet d'envisager la caractérisation des indices significatifs du point de vue du sujet impliqué dans l'action, qui relèvent d'un niveau pré-réfléchi de conscience, et qui participent à l'organisation des décisions. Elle constitue pour le chercheur, un moyen de comprendre la logique intrinsèque de la conduite du sujet qui a vécu l'expérience, telle qu'elle est verbalisée en entretien d'explicitation. »

Caroline Ganière et Marc Cizeron (université de Clermont Ferrand), en utilisant des techniques d'autoscopie et d'auto-explicitation en première, deuxième et troisième personne auprès de gymnastes, font un travail d'explicitation de l'aspect phénoménal de l'« intensité » de l'expérience corporelle. Le résultat identifie une certaine présence phénoménale de soi agissant et ressentant, c'est-à-dire la conscience d'un JE agent de son action. La granularité intentionnelle de l'action motrice et la présence intentionnelle de l'action motrice s'expriment dans la production par le sujet lui-même d'un dessin vertigineux. Ainsi est constitué un support pour l'explicitation, en facilitant l'identification de moments saillants, de sensations et d'existences particulières. 
Tableau 1.

\begin{tabular}{|c|c|c|c|c|}
\hline & Activité & Vécu corporel & Somatechniques & Références \\
\hline $\begin{array}{c}\text { Alain } \\
\text { Mouchet }\end{array}$ & $\begin{array}{c}\text { Dynamique } \\
\text { attentionnelle }\end{array}$ & $\begin{array}{l}\text { L'attention en } \\
1^{\text {re }} \text { personne }\end{array}$ & Psycho-phénoménologie & $\begin{array}{l}\text { P. Vermersch, E. Husserl, } \\
\text { J.P. Petit, H. Ripoll }\end{array}$ \\
\hline $\begin{array}{c}\text { Marc Cizeron } \\
\text { Caroline Ganière }\end{array}$ & $\begin{array}{l}\text { Habileté motrice } \\
\text { Gymnastique }\end{array}$ & $\begin{array}{l}\text { Graphe expérientiel } \\
\text { Autoscopie }\end{array}$ & $\begin{array}{l}\text { Explicitation en } 1^{\mathrm{er}}, \\
2^{\mathrm{e}} \text { et } 3^{\mathrm{e}} \text { personne }\end{array}$ & $\begin{array}{l}\text { P. Vermersch, P. Ricoeur, } \\
\text { M. Merleau-Ponty }\end{array}$ \\
\hline $\begin{array}{l}\text { Philippe Beaudoin } \\
\text { Christine Pépin }\end{array}$ & $\begin{array}{c}\text { Danse } \\
\text { intégrée }\end{array}$ & $\begin{array}{l}\text { Intimité } \\
\text { et Proximité }\end{array}$ & $\begin{array}{c}\text { Hybridation } \\
\text { valides/handicapés }\end{array}$ & $\begin{array}{l}\text { R. Huesca, J. Kerouanton, } \\
\text { M.C. Brown, } \\
\text { B. Curtis, A. Ptashek }\end{array}$ \\
\hline $\begin{array}{l}\text { Michel Récopé } \\
\text { Hélène Fache, } \\
\text { Simon Boyer, } \\
\text { Géraldine } \\
\text { Rix-Lièvre }\end{array}$ & $\begin{array}{l}4 \text { registres } \\
\text { kinesthésie } \\
\text { et sensations } \\
\text { Volley ball }\end{array}$ & $\begin{array}{l}\text { Sensibilité } \\
\text { écologique }\end{array}$ & $\begin{array}{c}\text { Sensibilités } \\
\text { à la proximité } \\
\text { et à la rupture }\end{array}$ & $\begin{array}{c}\text { G. Canguilhem, } \\
\text { E.A. Di Paolo, } \\
\text { N. Bernstein, F. Varela, } \\
\text { Y.-M. Visetti, } \\
\text { V. Rosenthal }\end{array}$ \\
\hline Nancy Midol & $\begin{array}{c}\text { Danse } \\
\text { improvisée }\end{array}$ & Awareness & La transe & $\begin{array}{c}\text { J.H. Austin, E. Collot, } \\
\text { B. Hell, B. Grison, } \\
\text { A.R. Damasio }\end{array}$ \\
\hline Yannick Vanpoulle & $\begin{array}{c}\text { Intelligence } \\
\text { motrice }\end{array}$ & Enaction & Autopoièse & $\begin{array}{l}\text { F. Varela, J.J. Temprado, } \\
\text { J. Paillard, C. PetitMengin }\end{array}$ \\
\hline $\begin{array}{l}\text { Thi Bich-Ngoc } \\
\text { Doan }\end{array}$ & $\begin{array}{c}\text { Arts } \\
\text { martiaux }\end{array}$ & $\begin{array}{l}\text { Expérience } \\
\text { directe }\end{array}$ & Perception & $\begin{array}{c}\text { S. Dehaene, J.P. Lachaux, } \\
\text { S. Gallagher, M. Bitbol, } \\
\text { C. Petitmengin }\end{array}$ \\
\hline Nicolas Burel & $\begin{array}{c}\text { Le genre } \\
\text { professionnel }\end{array}$ & $\begin{array}{l}\text { Intercorporalité } \\
\text { émotionnelle }\end{array}$ & $\begin{array}{l}\text { Toucher } \\
\text { Coping }\end{array}$ & C. Cherniss, Y. Clot \\
\hline $\begin{array}{l}\text { Bernard } \\
\text { Andrieu }\end{array}$ & Écologie pré-motrice & $\begin{array}{l}\text { Empathie } \\
\text { Inconsciente } \\
\text { du corps }\end{array}$ & $\begin{array}{l}\text { Techniques } \\
\text { d'activation }\end{array}$ & $\begin{array}{c}\text { B. Libet, K. Popper, } \\
\text { P. Parlebas, A. Berthoz, } \\
\text { D. Delignières, } \\
\text { J. Le Boulch, A. Noé }\end{array}$ \\
\hline
\end{tabular}

Renverser la situation de motricité classique, comme avec la danse intégrée présentée ici par Philippe Beaudoin et Christine Pépin (université de Lorraine), n'est pas une simple critique des normes esthétiques et du genre. Avec la danse intégrée, le handicap ne représente pas qu'une contrainte, source de nouveauté parmi d'autres. Cette démarche de danse basée sur la notion de contrainte n'apprend pas un mouvement, mais génère du mouvement à partir de l'expérience personnelle et des sensations du pratiquant.

Michel Récopé, Hélène Fache, Simon Boyer et Géraldine Rix-Lièvre (université Blaise Pascal, Clermont-Ferrand) mettent en œuvre une «éthologie phénoménologique fondée sur des postulats énactifs » qui permet de décrire l'activité de deux micro-identités de pratiquants de volley-ball. Cette approche du vécu corporel utilise une phénoménologie de l'action en situation et implique une étude écologique de la situation motrice de l'individu. La micro-identité de ces pratiquants est caractérisée par une cohérence globale relevant d'une sensibilité à la proximité du ballon, d'une sensibilité à l'enjeu de rupture de l'échange. Quatre registres sont alors distinguables. Le registre le plus global de l'activité et du vécu corporel définit un cadre d'interactions organisées par un sens global et un corps sensible. Le registre de la contextualisation de l'activité et du vécu corporel précise un cadre d'interactions organisées par un sens tactique et un corps de rationalité pratique. Le registre de la visée par déviation produit un cadre d'interactions organisées par un sens d'efficacité mécanique et un corps instrument de la précision. Le registre postural de l'activité et du vécu corporel implique un cadre d'interactions organisées par un sens kinesthésique et un corps sensitif.

Nancy Midol (université de Nice) à travers des exemples vécus et racontés en première personne, parle « d'une mise en mouvement du corps, lors d'une danse improvisée en public et du surgissement impromptu de quelque chose qui dépasse et déjoue la conscience du danseur. La perte du contrôle apparaît comme une forme de dépossession de soi qui correspond dans d'autres sociétés au phénomène de possession de soi par une entité étrangère. La manifestation de cela s'impose, l'être, en dépit de sa volonté et de son projet, n'a plus la maîtrise de ses comportements, il est agi par.»

Yannick Vanpoulle (université de Lyon) interroge la notion d'intelligence motrice dans les modèles de l'autoorganisation et de la complexité pour terminer par des 
modèles holistiques La connaissance par corps est bien globale et dépendante des différents niveaux d'organisation et des interactions différenciées avec l'environnement. Elle est une cognition se manifestant par l'émergence de propriétés permettant des solutions adéquates pour des catégories de tâches données.

Thi Bich-Ngoc Doan (université Panthéon-Sorbonne) abaisse le seuil de conscience en partant d'une perception sans conscience. L'unité corps-esprit, la perception globale du corps, la fusion ou la connexion avec le corps du partenaire ou l'environnement, sont des caractéristiques de cette perception qui s'unifie, perd en quelque sorte ses limites corporelles et mentales pour s'ouvrir à une perception énergétique plus large. Les théories et les résultats expérimentaux sur la conscience pure, la perception directe et les neurones miroirs, la vitesse de traitement de l'information, la frontière conscient/non conscient, le contrôle attentionnel léger, l'action naturelle, sont des pistes qui pourraient être étudiées plus en détail afin de juger de l'utilité et de la pertinence de les relier plus étroitement avec des données en première personne sur la vitesse de perception, l'absence de réflexion, la perception immédiate, l'action spontanée sans conscience.

Pour Nicolas Burel (université de Strasbourg) l'expérience soma-esthétique du toucher de l'enseignant d'Education Physique et Sportive sur l'élève est envisagée comme l'opportunité de resituer les construits de la psychologie positive et de la psychologie du stress dans une écologie pré-motrice sous-jacente, et d'ouvrirainsi un nouveau champ d'investigation entre affectivité du vivant, affectivité vécue, et affectivité décrite. Elle se fonde sur l'hypothèse du développement tacite de compétences émotionnelles, vue sous l'angle psychophénoménologique.

Pour Bernard Andrieu (université de Lorraine) l'écologie pré-motrice pose la question de l'interaction entre le cerveau et la conscience : le cerveau se sertil de la conscience, si la question n'était trop finaliste, pour se faire oublier là où la conscience ne peut en aucune façon l'apercevoir? Comme il ne peut être perçu dans le temps réel et vivant de son activité, le cerveau émerge dans sa conscience dans une rémanence neurocognitive dont l'origine est inaccessible dans le présent de la conscience. Nous n'avons conscience que du cerveau passé car pendant que nous en avons conscience le cerveau vivant lui-même continue déjà à anticiper la réponse motrice. Cette rémanence du temps de la conscience n'interdit pas à la phénoménologie de décrire le contenu de la conscience en première personne. Mais la conscience en première personne aussi subjective soitelle n'est pas le cerveau du corps vivant en première personne! Le cerveau se sait-il lui-même en première personne indépendamment de la conscience qui ne peut le percevoir qu'avec retard? 